\title{
Incorporating biomarkers into clinical trials in cardiovascular medicine
}

\author{
This article was published in the following Dove Press journal: \\ Current Biomarker Findings \\ 4 September 2014 \\ Number of times this article has been viewed
}

\author{
Hsiu-Fang Lee \\ Sho Ota \\ Trialynx, Inc., Ann Arbor, MI, USA
}

Correspondence: Sho Ota

Trialynx, Inc., 24 Frank Lloyd Wright

Drive Box 36I Ann Arbor, MI,

USA 48105

Tel + I 7342749257

Email sota@trialynx.com

\begin{abstract}
Developing novel therapies and establishing clinical benefits over conventional therapies has become a highly competitive and a costly endeavor. In this regard, biomarkers have become increasingly important in accurately assessing both efficacy and safety of these novel therapies. While the use of biomarkers has proved vital in cardiovascular research and disease management, current established biomarkers are inadequate in capturing and accounting for the entire cardiovascular disease process and responses to therapeutic interventions. Substantial effort has been made in exploring and clinically evaluating novel biomarkers. The present review aims to shed light on some of the novel cardiovascular biomarkers being investigated, including high sensitivity C-reactive protein, proprotein convertase subtilisin/kexin type 9, P-selectin, secretory phospholipase $\mathrm{A}_{2}$, lipoprotein-associated phospholipase $\mathrm{A}_{2}$, and growth differentiation factor 15, and illustrate their relevance to clinical research.
\end{abstract}

Keywords: hs-CRP, PCSK9, P-selectin, sPLA, Lp-PLA, GDF-15

\section{Introduction}

Significant strides have been made in cardiovascular medicine over the past 50 years. According to the National Vital Statistics Report, "diseases of heart" is one of only two major causes of death with a steady decline in death rate since 1958 in the United States, with the other being cerebrovascular disease. ${ }^{1}$ In contrast, death rates of other major causes of death have been either remained stagnant or increased. And yet, cardiovascular disease continues to be the leading cause of death in the US, accounting for $24.2 \%$ of all mortalities. Despite the wide array of highly effective treatments available for cardiovascular diseases, further research is clearly needed to address unmet needs by better understanding the optimal use of current therapies and developing novel therapies.

In clinical research, biomarkers play a crucial role in gathering efficacy and safety data, especially when study duration and sample size is limited. Biomarker is defined by the National Institutes of Health as "a characteristic that is objectively measured and evaluated as an indicator of normal biologic processes, pathogenic processes, or pharmacologic responses to a therapeutic intervention."2 Biomarkers may be physical characteristics such as blood pressure, heart rate, and waist circumference, or biochemical characteristics such as serum cholesterol, breath alcohol, and urine albumin. Furthermore, a "valid" biomarker is defined by the Food and Drug Administration pharmacogenomics guidance as:

a biomarker that is measured in an analytical system with well-established performance characteristics and for which there is an established scientific framework or body of evidence that elucidates the physiologic, toxicologic, or clinical significance of the test results. ${ }^{3}$ 
In order for a biomarker to be valid, and therefore practical, certain criteria must be met. For one, reference limits must be clearly established so that any abnormality can be clearly captured. In addition, the biomarker must have limited interindividual variability so that the reference limits are applicable across the entire target population. Thirdly, any prognostic or diagnostic ability of a biomarker must have some added value to currently available methods, whether it is improved accuracy, reliability, speed, or cost. Also, changes to the biomarker must have an associated clinical significance such that the change leads to a change in treatment or treatment dose. Establishing a valid biomarker requires intensive research and clinical trials to prove that the above criteria are met. However, biomarkers offer significant benefits not only in clinical practice, but also in clinical trials and are now indispensable tools in clinical research.

In clinical trials, biomarkers may be used for a wide variety of purposes. For example, biomarkers allow investigators to screen for patients that most likely benefit from an investigational therapy. In the development of Herceptin $^{\text {TM }}$ ([trastuzumab]; Genentech, Inc., San Francisco, CA, USA), an antibody against human epidermal growth factor receptor 2 (HER2) for the treatment of breast cancers, investigators recruited only patients with HER2-positive breast cancer to maximize the efficacy of the investigational drug. ${ }^{4}$ Alternatively, measuring biomarkers at select time points allow investigators to predict long-term clinical outcomes in patients. The Framingham risk assessment is one such biomarker that allows the prediction of the 10-year risk of having a heart attack based on a one-time assessment of biomarkers including age, gender, cholesterol levels, smoking status, and blood pressure. ${ }^{5}$ In addition, biomarkers may detect rare safety concerns in clinical trials that would otherwise not present clinically without a large sample size or a long follow-up period. For all clinical trials investigating novel drugs, monitoring the QTc intervals in electrocardiograms has become a standard for identifying risks of fatal cardiac arrhythmias. ${ }^{6}$ Drugs that increase the QTc interval raise concerns for fatal ventricular tachycardia as a possible side effect. As illustrated in the examples above, appropriate use of validated biomarkers in clinical trials offers innovative approaches in gathering much needed efficacy and safety data with limited resources and study duration.

The current challenge in the utilization of biomarkers in cardiovascular research, however, is that currently validated biomarkers do not accurately predict clinical outcomes in a significant proportion of patients. ${ }^{7}$ Approximately 40\% of patients with below-average low-density lipoprotein cholesterol (LDL-C) still die today from cardiovascular diseases, despite LDL-C being widely recognized as a key clinical measure and target of therapy for improving cardiovascular outcomes. Cardiovascular disease is a multifaceted disease that involves disease processes such as atherosclerosis, myocardial disorders, and blood disorders. Therefore, one biomarker should not be expected to reflect the variety of biological processes that are involved in cardiovascular disease. Furthermore, many of the biomarkers used in cardiovascular research are affected by a wide range of factors including age, gender, race, pregnancy status, use of medications, diet, exercise, time of day, and fasting state. ${ }^{8}$ With so many compounding factors affecting these biomarkers, the use of these biomarkers for the assessment of treatment efficacy or safety can be highly suspect in clinical settings where not every one of these factors can be controlled. While the use of biomarkers has proved vital in cardiovascular research so far, novel biomarkers are clearly needed to better identify the therapeutic needs and benefits not captured by currently available biomarkers. To address this concern, the present review aims to shed light on some of the novel cardiovascular biomarkers, including high sensitivity C-reactive protein (hs-CRP), proprotein convertase subtilisin/kexin type 9 (PCSK9), P-selectin, secretory phospholipase $\mathrm{A}_{2}\left(\mathrm{sPLA}_{2}\right)$, lipoprotein-associated phospholipase $\mathrm{A}_{2}\left(\mathrm{Lp}-\mathrm{PLA}_{2}\right)$, and growth differentiation factor 15 (GDF-15), and illustrate their relevance to clinical practice and research.

\section{hs-CRP}

hs-CRP has gained much attention as a potential biomarker for assessing the risk of a recurrence of a cardiovascular event in patients who have experienced myocardial ischemia ${ }^{9}$ Although C-reactive protein (CRP) is an acute phase reactant, the chronic levels that are present at low concentrations as measured by hs-CRP are thought to predict clinical outcomes in these patients. CRP has a plasma half-life of 19 hours, and the concentration of hs-CRP can range anywhere from less than $1 \mathrm{mg} / \mathrm{L}$ to $3 \mathrm{mg} / \mathrm{L}$ or greater. CRP is produced predominantly by hepatocytes in response to cytokines, with interleukin 6 (IL-6) being the most potent of the cytokines. IL-6, on the other hand, is released by leukocytes in response to vascular smooth muscle damage, including atherosclerosis. Therefore, the level of CRP is thought to reflect the amount of damage and atherosclerosis on the vasculature.

The function of CRP in heart disease is very complex and is not yet fully understood. CRP is a mediator of inflammatory pathways and is thought to contribute to the 
development of atherosclerosis via macrophage polarization and facilitation of monocyte adhesion and transmigration into the vessel wall. ${ }^{7}$ In addition, CRP is thought to bind and recruit LDL-C to the sites of vascular injury. The clinical evidence, on the other hand, for the use of hs-CRP as a predictor for the recurrence of a cardiovascular event is numerous. In the Cholesterol and Recurrent Events (CARE) trial, use of pravastatin in patients post-myocardial infarction (MI) showed significant reduction in the level of CRP and the risk of a cardiovascular event, regardless of the changes in LDL. ${ }^{9}$ The study result showed a $75 \%$ higher relative risk of recurrent events in patients with CRP levels in the highest quintile compared to those in the lowest quintile (relative risk $=1.77, P=0.02$ ). In the Multiple Risk Factor Intervention Trial (MRFIT), a randomized prospective trial investigating the effect of a multifactor intervention program in the primary prevention of heart disease in high-risk men, a significant correlation was observed between hs-CRP and cardiovascular mortality over an average follow-up period of 7 years. ${ }^{10}$ The correlation was observed not just in men, but also in women in the Women's Health Study (WHS). ${ }^{11}$ The study found that, in healthy women, elevated hs-CRP was a strong predictor of cardiovascular events with a relative risk of 2.0, compared to only 1.3 for elevated LDL-C. While the exact molecular interaction by which CRP exerts its effect is yet to be clarified, the clinical evidence does suggest that CRP is a potentially useful biomarker in cardiovascular medicine and research.

Unfortunately, the validity of hs-CRP as a predictive biomarker has not been consistent in clinical trials. In the Justification for the Use of Statins in Prevention: An Intervention Trial Evaluating Rosuvastatin (JUPITER) trial, the study established that the use of statins in patients with low LDL still provide significant mortality benefit among patients with elevated hs-CRP $(>2 \mathrm{mg} / \mathrm{L}) .{ }^{12}$ However, the study was criticized for not having a low hs-CRP group, and therefore not being able to determine whether hs-CRP levels contributed to the observed effect of rosuvastatin. ${ }^{13}$ Furthermore, post hoc analysis of the study data was not able to detect any correlation between hs-CRP levels and statin response. ${ }^{14}$ In the Prospective Study of Pravastatin in the Elderly at Risk (PROSPER) trial, no correlation was observed between hs-CRP and response to statin therapy or the risk of future cardiovascular event. ${ }^{15}$ The findings of the PROSPER trial were further supported in the Heart Protection Study (HPS) trial, which demonstrated reduction in cardiovascular events with statin use regardless of hs-CRP levels. ${ }^{16}$ As a result, monitoring hs-CRP levels has not yet been accepted as part of routine procedures in treating cardiovascular diseases.

The reasons for the inconsistency in the findings across so many clinical trials may be due to the fact that hs-CRP is affected by a multitude of cardiovascular and noncardiovascular factors. ${ }^{17}$ For example, the baseline level of hs-CRP appears to vary across ethnic groups, with the highest levels observed in African Americans, followed by Hispanics, south Asians, whites, and the lowest in east Asians. Furthermore, women tend to have higher baseline hs-CRP compared to men, and hs-CRP increases with age regardless of the presence of cardiovascular disease. Additionally, adipose tissue can be a significant source of IL-6, which stimulates CRP production and affects baseline levels of hs-CRP. ${ }^{18}$ In fact, a clinical trial has found that the reliability of hs-CRP as a predictor of cardiovascular events is significantly lower in obese patients. ${ }^{19}$ Other factors known to influence hs-CRP levels include age, diabetes, hypertension, oral contraception, exercise, alcohol use, periodontal disease, diet, environmental pollutants, and smoking. ${ }^{7}$ Moreover, hs-CRP can have significant day-to-day variations. An analysis of the data from the National Health and Nutrition Examination Survey (NHANES), a survey conducted by the Centers for Disease Control, has found that CRP can change by an average of $46.2 \%$ over a 19 -day period. ${ }^{20}$ Of the patients classified as elevated in the first measurement, $32 \%$ of patients were within normal limits after 19 days. To further complicate the matter, $50 \%$ of all adults and $41 \%$ of 20 -year-olds have an elevated hs-CRP level ( $>2 \mathrm{mg} / \mathrm{L}) .{ }^{17}$ The appropriate use of hs-CRP is still unknown due to the high variability observed across many clinical trials.

Because of the lack of a clear consensus in the findings across clinical trials, the expert panel for the US Preventive Services Task Force recommended against routine testing of hs-CRP for primary prevention of cardiovascular disease in 2002. ${ }^{21}$ The panel did recommend, however, testing in intermediate-risk patients with a 10 -year risk of $10 \%-20 \%$. This recommendation is supported by observations in clinical trials where patients with atherosclerosis and a baseline hs-CRP of $3 \mathrm{mg} / \mathrm{L}$ had a $50 \%$ higher likelihood of experiencing a future cardiovascular event compared to those with a baseline hs-CRP of less than $1 \mathrm{mg} / \mathrm{L}{ }^{7}$ This finding highlights the importance of not utilizing hs-CRP as an independent risk factor, but rather as a supplement to other more established cardiovascular biomarkers such as LDL, blood pressure, and others. At the moment, the appropriate use of hs-CRP in clinical trials remains uncertain other than as a biomarker for inflammatory activity. Much research is still needed to 
establish its diagnostic or prognostic capabilities as clinical endpoints.

\section{PCSK9}

PCSK9 is an enzyme expressed by the liver, intestines, and the kidneys that plays a role in the degradation of LDL receptors in the liver. ${ }^{22}$ Upon binding to the membrane-bound LDL receptor, PCSK9 induces a lysosomal destruction of the LDL receptor, thereby reducing the ability of the liver to uptake and metabolize LDL from circulation. PCSK9 was first discovered through a genetic analysis of patients with familial hypercholesterolemia, which found that a gain of function mutation strongly correlated with hypercholesterolemia. A loss of function mutation correlated with lower incidence of cardiovascular diseases. PCSK9 is involved in the accumulation of triglycerides in visceral adipose tissues as well. Expression of PCSK9 is activated primarily in response to low levels of intracellular cholesterol, but can also be stimulated by other factors such as insulin. Conversely, PCSK9 expression is downregulated in response to inflammation, LDL-C, and fasting. The half-life of PCSK9 is 5 minutes, but may be longer in the absence of LDL receptors. PCSK9 is being investigated not only as a biomarker, but also as a therapeutic target for the treatment of hypercholesterolemia. Inhibition of PCSK9 would allow increased expression of LDL receptors in the liver, thus increasing the metabolism of circulating LDL-C. Since its discovery in 2003, PCSK9 has been studied extensively to establish its clinical relevance and many clinical trials are underway to develop agents against PCSK9.

With no therapeutic agents currently on the market to effectively target PCSK9, the majority of the clinical evidence behind the use of PCSK9 as a biomarker is derived from case studies in patients with mutations of the gene. ${ }^{23}$ In the Atherosclerosis Risk in Communities (ARIC) trial, a retrospective trial involving over 12,000 patients, the loss of function mutation of the PCSK9 gene was found to be associated with a $28 \%$ reduction of LDL-C and $88 \%$ reduction in the risk of heart disease among African Americans, and a 15\% and a $47 \%$ reduction, respectively, among whites. ${ }^{24} \mathrm{~A}$ similar reduction in LDL-C levels was observed even among children with the mutations in the Bogalusa Heart Study. ${ }^{25}$ Interestingly, one of the most popular classes of cardiovascular medications, statins, has been found to upregulate both PCSK9 expression and LDL receptors in a dose- and potency-dependent manner. Administration of atorvastatin $10 \mathrm{mg}$ and $40 \mathrm{mg}$ has been reported to increase plasma PCSK9 levels by $7.4 \%$ and $34 \%$, respectively. ${ }^{26} \mathrm{~A}$ more potent statin, rosuvastatin $20 \mathrm{mg}$, has been reported to increase PCSK9 levels by $28 \%-35 \% .{ }^{27}$ The increased expression of PCSK 9 in response to statin therapy likely attenuates the therapeutic benefit of statins on LDL-C reduction. Therefore, combining a PCSK9 inhibitor with statin use is expected to have a synergistic effect in lowering LDL through upregulation of LDL receptors without increasing PCSK9 activity. The effects of other lipid lowering agents, such as fibrates and ezetimibe, on PCSK9 expression level has been inconclusive so far. ${ }^{22}$ These positive findings have stimulated intense efforts to develop novel inhibitors of PCSK9 as a treatment option for cardiovascular diseases, as evidenced by over 20 clinical trials involving PCSK9 at the time of writing the current review.

\section{P-selectin}

$\mathrm{P}$-selectin is a cell adhesion molecule that mediates the initial recruitment of leucocytes to the inflammatory site. ${ }^{28}$ When platelets or endothelial cells are activated by stimuli (eg, inflammatory cytokines, thrombin, histamine or oxidized LDL), P-selectin is translocated to the cell surface. Once migrated to the cell surface, $\mathrm{P}$-selectin binds to $\mathrm{P}$-selectin glycoprotein ligand-1 (PSGL-1) and sialyl-Lewis ${ }^{\mathrm{x}}$, which are constitutively expressed in all leukocytes. The interaction between P-selectin and PSGL-1 serves as the point of initiation of the interaction between platelets, endothelium, and leukocytes. The expression level and degree of glycosylation of PSGL-1 may differ between subtypes of leukocytes, which render endothelial selectivity in recruiting different subtypes of leukocytes to lesions. ${ }^{29}$

P-selectin has two functional forms: membrane-bound P-selectin on platelets and endothelial cells, and soluble $\mathrm{P}$-selectin (sP-selectin) circulating in the blood. sP-selectin is a truncated form of P-selectin, lacking the transmembrane and intracellular domains. The origin of sP-selectin includes platelets and endothelial cells via cleavage of the membranebound P-selectin, as well as direct expression. ${ }^{30}$ As with membrane-bound $\mathrm{P}$-selectin, $\mathrm{P}$-selectin has been shown to exert proinflammatory, ${ }^{31,32}$ prothrombotic, ${ }^{33}$ and procoagulant activities. ${ }^{34}$ Elevated levels of $\mathrm{sP}$-selectin are seen in patients with vascular diseases, ${ }^{31,35}$ hypercholesterolemia, ${ }^{36}$ hypertension, ${ }^{37}$ unstable angina, ${ }^{38}$ exuberated stroke, ${ }^{39}$ and aortic valve stenosis. ${ }^{40}$

P-selectin has been demonstrated to be a key initiator in the development of atherosclerosis via the formation of platelet-monocyte aggregates (PMA). ${ }^{41-43}$ P-selectin on the surface of activated platelets binds PSGL-1 on the monocytes to form PMA. PMA binds to the endothelium at a higher affinity than monocytes and delivers cytokines 
and procoagulant microparticles (MPs) at the site of inflammation in the vasculature. This results in upregulation of procoagulant tissue factors that may spur fibrin deposition and thrombus formation. ${ }^{44}$ As expected, elevated PMA levels are seen in acute coronary syndrome (ACS) patients ${ }^{45}$ and increase the risk of cardiac events in non-ST segment elevation MI patients. ${ }^{46,47}$

Another critical role of P-selectin in atherosclerosis is the prerequisite step of leukocyte recruitment through a dynamic adhesion cascade that includes tethering, rolling, firm adhesion, and transmigration of leukocytes into the endothelium. ${ }^{48-52} \mathrm{P}$-selectin expressed on stimulated endothelial cells mediates leukocyte tethering and rolling by binding to PSGL-1 present on the leukocyte surface. ${ }^{53} \mathrm{P}$-selectin acts as the braking process to decelerate fast-flowing leukocytes in the blood stream. Decelerated leukocytes roll along and interact with the endothelium to further stimulate an inflammatory response, including expression of other adhesion molecules, such as intercellular adhesion molecule-1 and vascular cell adhesion molecule- $1 .{ }^{54}$ Once firm adhesion is achieved through these adhesion molecules, leukocytes infiltrate into the arterial vessel wall to initiate the formation of the atherosclerotic plaque.

$\mathrm{P}$-selectin is also involved in thrombus formation. Interaction between P-selectin and its ligand is essential for thrombus formation in two ways: P-selectin on the activated platelets in the thrombus tethers monocytes, and sP-selectin binds to PSGL-1 on leukocytes to induce the release of MPs. ${ }^{34}$ Through P-selectin-PSGL-1 binding, the activated platelets in thrombi recruit and activate monocytes, much like in the case with PMA. Additionally, MPs are recruited to the thrombi by the interaction between P-selectin on the activated platelets in thrombi and PSGL-1 on the MPs, which further stimulates coagulant and inflammatory mechanisms. ${ }^{55,56}$ Through tethering of monocytes and generation of MPs, P-selectin mediates thrombus formation and ultimately contributes to arterial occlusion and ischemia.

Increased expression of P-selectin is observed in a variety of cardiovascular diseases, including peripheral artery diseases, ACS, and stroke. Elevated levels of P-selectin have been observed on the endothelial cells overlying atherosclerotic plaques ${ }^{57}$ and on circulating platelets in patients with peripheral arterial diseases. ${ }^{58,59}$ Patients with unstable angina, congestive heart failure, or acute MI show significantly elevated levels of sP-selectin and platelet-bound P-selectin as compared to healthy subjects. ${ }^{60}$ In addition, patients with unstable angina exhibit significantly higher levels of P-selectin expression in coronary atherectomy specimens than patients with stable angina. ${ }^{61,62}$ Elevated levels of $\mathrm{sP}$-selectin were also observed in patients with vascular disorders such as hypertension and hypercholesterolemia. ${ }^{36,37}$ In patients with acute cerebral infarction, P-selectin and sP-selectin levels have been shown to be elevated compared to healthy individuals, and to correlate with an increase in phospholipase $\mathrm{A}_{2}$ (PLA). ${ }^{63,64}$ Taken together, P-selectin appears to have a correlation not only with onset of disease, but also with progression of disease and shows great promise as a cardiovascular biomarker.

Because of the association between escalating levels of sP-selectin and pathological states, sP-selectin is being investigated as a diagnostic biomarker for cardiovascular diseases ${ }^{65,66}$ For instance, plasma sP-selectin levels may be an indicator for successful thrombolysis in acute MI. ${ }^{66}$ Acute MI elevates P-selectin levels by approximately fourfold, but diminishes within 24 hours after successful thrombolytic therapy using tissue plasminogen factor. In contrast, sP-selectin levels remain high in patients with unsuccessful thrombolysis. Similarly, cell surface P-selectin and plasma sP-selectin have been suggested as potential markers of platelet activation in a number of disease models, such as ischemic heart disease, rheumatic mitral stenosis, and atrial fibrillation. ${ }^{67}$

P-selectin may not only predict disease outcome and progress, but also cardiovascular complications following therapeutic interventions. Plasma sP-selectin levels are elevated in patients with myocardial ischemia, and further rise after reperfusion therapy by nearly $30 \%$ over a 1-week period post-reperfusion. ${ }^{68}$ This increase leads to PLA formation, and is believed to significantly contribute to post-angioplasty restenosis. Furthermore, P-selectin levels rise post-revascularization in the heart, which is thought to reflect the extent of reperfusion injury caused by the therapeutic intervention. ${ }^{69,70}$ In support of this notion, inhibition of P-selectin via administration of recombinant soluble P-selectin glycoprotein ligand-Ig in a swine model of reperfusion post-myocardial-ischemia was able to maintain myocardial blood flow at a pre-ischemia level for 2 hours post reperfusion. ${ }^{70}$ In contrast, only $65 \%$ of the myocardial blood flow was maintained in the control group $(P<0.05)$. In addition, P-selectin inhibitors have demonstrated a significant increase in endothelium-dependent vasorelaxation of coronary arteries, reduction in infarct size, and reduction in the impairment of ejection fraction in various animal models of MI. ${ }^{70-72}$ In addition to playing a complimentary role in assessing cardiovascular disease state, P-selectin also may have a niche role in monitoring complications post-intervention, 
including impeding atherosclerosis development, preventing thrombus growth and fibrin deposition, ${ }^{42,73-75}$ alleviating myocardial reperfusion injury, ${ }^{43,76}$ and reducing restenosis. ${ }^{77}$

Intriguingly, a number of currently available therapies are known to have effects on P-selectin, and may explain some of the therapeutic benefits observed with these agents beyond their intended effect. In a clinical trial involving 148 patients, patients taking $100 \mathrm{mg}$ aspirin showed significantly lower plasma sP-selectin levels post-percutaneous coronary intervention (PCI) after a loading dose of $600 \mathrm{mg}$ clopidogrel, compared to those not on aspirin $(P=0.004) .{ }^{78}$ The presence of the CYP2C19*2 allele, which is responsible for clopidogrel metabolism, had no impact on the inflammatory markers in these patients, suggesting clopidogrel did not influence sPselectin levels. Heparin and its analogues are also known to inhibit P-selectin activity. ${ }^{79}$ In an in vitro experiment using blood of healthy volunteers, heparin at therapeutic plasma concentrations suppressed P-selectin expression on the surfaces of thrombin-stimulated platelets by nearly $80 \%$ $(P<0.001)$, and enoxaparin did so by approximately $60 \%$ $(P=0.0014) .{ }^{79}$ The inhibitory action is thought to be mediated through inhibition of $\mathrm{P}$-selectin binding to sialyl-Lewis ${ }^{\mathrm{x}}{ }^{80}$ Attempts have been made to produce a heparin analogue without the anticoagulant effect for P-selectin inhibition. ${ }^{81}$ Statins are another class of therapeutic agents known to inhibit P-selectin activity. In a crossover trial involving 32 patients with atherosclerotic ischemic stroke, platelet P-selectin level was significantly lower after 12 weeks of treatment with simvastatin $20 \mathrm{mg}$, compared to the levels after a 12-week washout period. ${ }^{82}$ The reduction in $\mathrm{P}$-selectin expression was independent of changes in LDL. The author proposed that the decrease in P-selectin level might be attributable to a statin-mediated anti-inflammatory mechanism, as evidenced by reduced CRP levels. In another clinical trial involving coronary artery disease (CAD) patients, patients receiving statins for 6 months had approximately 30\% lower plasma sP-selectin levels compared to those not on statins $(P<0.0001) .{ }^{83}$ The sP-selectin reduction was accompanied by over 50\% reduction in hs-CRP levels $(P<0.001)$. Although the exact mechanism behind statin-induced inhibition of P-selectin is yet to be elucidated, the findings above suggest targeted inhibition of P-selectin, in combination with current standard of care, may prove to be a novel and effective treatment for cardiovascular diseases.

\section{PLA}

Two members of the PLA enzyme family, sPLA 2 and Lp$\mathrm{PLA}_{2}$, have been evaluated as biomarkers of cardiovascular risk in population-based studies inclusive of apparently healthy individuals and patients with established coronary heart disease (CHD) such as ACS and MI ${ }^{84}$ PLAs are capable of generating various lipid mediators by modification of LDL-C, and are thought to mediate vascular inflammation and atherosclerosis. Lp-PLA 2 and SPLA $_{2}$ are molecularly and biochemically distinct and thus their roles and underlying mechanisms of action in the blood and within the vascular wall are likely to be different. ${ }^{85,86}$ The prognostic values of circulating levels of Lp-PLA ${ }_{2}$ and SPLA $_{2}$ have been intensively investigated and they have been suggested as independent predictors of cardiovascular events in various patient populations, which will be further discussed below.

\section{Lp-PLA}

Although still controversial, Lp-PLA 2 is thought to contribute to the progression of atherosclerosis. Lp-PLA $A_{2}$ is predominantly bound to LDL in humans. ${ }^{87,88} \mathrm{~A}$ recent study on hypercholesterolemic minipigs showed that elevated Lp$\mathrm{PLA}_{2}$ activity increased levels of lysophosphatidylcholine, oxidized LDL, and activity of proinflammatory genes in coronary plaque macrophages, which hasten the progression of atherosclerosis. ${ }^{89}$

The suggestion of Lp-PLA 2 as a predictor for cardiovascular diseases originated from the West of Scotland Coronary Prevention Study (WOSCOPS) in which each of the 580 men presenting with a coronary event (ie, nonfatal MI, death from $\mathrm{CHD}$, or a revascularization procedure) were matched for age and smoking status with two control subjects (1,160 controls in total) from the same cohort without a coronary event. ${ }^{90}$ Lp-PLA ${ }_{2}$, CRP, and fibrinogen levels were measured at baseline along with other traditional risk factors. The findings in this study showed a positive correlation between increased circulating Lp-PLA $\mathrm{A}_{2}$ levels and the risk of coronary events in hypercholesterolemic men even after accounting for age, systolic blood pressure, and lipoprotein levels. On the other hand, other traditional risk factors had weaker correlations after accounting for the aforementioned variables. The prognostic value of $\mathrm{Lp}_{\mathrm{PLA}}$ for cardiovascular events has also been demonstrated in patients with stable CAD. ${ }^{91-93}$ In addition to predicting the risk of a cardiac event, Lp-PLA $\mathrm{L}_{2}$ has been suggested as a risk predictor for restenosis. In a recent study involving 326 patients eligible for stent placement, elevated baseline levels of Lp-PLA 2 ( $>200 \mathrm{ng} / \mathrm{mL})$ were associated with an increased risk for restenosis after 1 year. ${ }^{94}$ Lp-PLA ${ }_{2}$ has been studied as a biomarker for a variety of cardiovascular diseases and may prove to be useful in both clinical practice and research. 
One caveat, however, is that Lp-PLA 2 has not been shown to be a reliable cardiovascular biomarker for patients with ACS. The data from the North Wuerttemberg and Berlin Infarction Study-II (NOBIS-II) study suggested that Lp$\mathrm{PLA}_{2}$ levels might predict cardiovascular complications only in a subgroup of ACS patient with moderately increased $\mathrm{N}$-terminal pro-brain natriuretic peptide (NT-proBNP). ${ }^{95}$ Another study in patients with MI reported a positive association between elevated levels of Lp-PLA 2 and 1-year cardiac mortality. ${ }^{96}$ However, subsequent large studies did not show such consistent findings as the aforementioned studies. A meta-analysis of Lp-PLA 2 levels in 1,362 ACS patients from the FRagmin and Fast Revascularization During Instability in Coronary Artery Disease (FRISC II) study, 904 ACS patients from the Global Use of Strategies to Open Occluded Arteries IV (GUSTO IV) study, and 435 apparently healthy controls showed no association between Lp-PLA 2 levels and recurrent MI or mortality. ${ }^{97}$ Similarly, analysis of Lp-PLA 2 levels from 3,648 ACS patients at admission and from 3,265 patients at 30 days after ACS in the PRavastatin Or atorVastatin Evaluation and Infection Therapy-Thrombolysis In Myocardial Infarction (PROVE IT-TIMI22) trial found no association with cardiovascular events. ${ }^{98}$ However, the patients with Lp-PLA 2 activity in the highest quintile at the 30-day follow-up did present increased risk of recurrent cardiovascular events compared to patients with Lp-PLA ${ }_{2}$ activity in the lowest quintile. Further adding questions to the prognostic value of Lp-PLA, the recent Phase III STabilisation of Atherosclerotic plaque By Initiation of darapLadIb TherapY (STABILITY) trial concluded that darapladib, a specific Lp-PLA ${ }_{2}$ inhibitor, failed to meet its efficacy endpoints in patients with stable CHD as defined by having at least one of the following: previous MI, previous PCI, previous coronary artery bypass graft, or multivessel CHD. ${ }^{99}$ In the 15,000-patient trial, darapladib failed to meet the primary endpoint of reduction in major adverse cardiovascular events (comprised of cardiovascular death, MI and stroke, or all-cause mortality). ${ }^{99}$ In the Stabilization of Plaques using Darapladib - Thrombolysis in MI 52 (SOLID-TIMI52) trial, darapladib also failed to meet the primary endpoint of a reduction of major coronary events in patients with ACS. ${ }^{100}$ As such, Lp-PLA activity has not been conclusive in its ability to predict clinical outcomes in patients with ACS.

Much work is still needed to fully understand Lp-PLA and its association with cardiovascular disease. For example, some of the studies mentioned above compare Lp-PLA activity level rather than the mass of circulating Lp-PLA, but not much is understood regarding which method of measurement more reliably predicts outcome. Although the prognostic value of Lp-PLA ${ }_{2}$ for cardiovascular risk has not been consistent among all populations, the currently available data suggest that measurement of Lp-PLA ${ }_{2}$ could improve cardiovascular risk assessment beyond traditional risk factors in diseased patients.

\section{sPLA}

In contrast to Lp-PLA ${ }_{2}$, the mechanism for $\mathrm{SPLA}_{2}$ in promoting atherosclerosis is more solid and conclusive. ${ }^{101}$ Circulating levels of SPLA $_{2}$ increase greatly during systemic inflammatory conditions. The hydrolytic action of $\mathrm{sPLA}_{2}$ generates various lipid mediators from the lipid deposits in the plaques on the arterial wall. The lipid mediators further trigger local inflammatory cellular response, which leads to atherosclerotic disease progression. In addition, $\mathrm{sPLA}_{2}$ may potentiate the binding and retention of LDL on the plaques by increasing the affinity of apolipoprotein B100 on LDL particles for glycoaminoglycans and proteoglycans on the arterial wall. Furthermore, $\mathrm{sPLA}_{2}$ is implicated in the production of isoprostanes, which exhibit strong mitogenic activity and induce platelet aggregation and vasoconstriction. Moreover, a study using C57BL/6 mice given a high-fat diet demonstrated that overexpression of sPLA $_{2}$ leads to increased susceptibility to atherosclerosis. ${ }^{101}$ These findings led to a host of clinical trials to elucidate the clinical significance of sPLA $_{2}$ as a cardiovascular biomarker.

The prognostic value of sPLA $_{2}$ has been addressed in a number of studies on apparently healthy subjects, ${ }^{102,103}$ patients with stable CAD, ${ }^{98,104-106}$ ACS patients, ${ }^{107,108}$ patients with unstable angina, ${ }^{109}$ and patients with acute MI. ${ }^{110}$ The European Prospective Investigation of Cancer (EPIC)-Norfolk Study, which was a two-nested case-control study, found that increased $\mathrm{SPLA}_{2}$ levels and activity correlated with the rate of first occurrence of a coronary event during 6 years of follow-up in relatively healthy individuals. ${ }^{102,103}$ In another study involving 142 patients with angiographically proven stable CAD, the presence of stable CAD was associated with higher sPLA ${ }_{2}$ levels compared to control individuals. ${ }^{104} \mathrm{In}$ the study by Koenig et al, increased sPLA ${ }_{2}$ levels after PCI were associated with subsequent $\mathrm{CAD}$ events. ${ }^{108}$ The utility of measuring $\mathrm{SPLA}_{2}$ activity was further supported by another large cohort in the PEACE study. ${ }^{111}$ The study, involving 3,708 patients with stable CAD, found that increased sPLA activity was associated with increased risk of adverse events such as cardiovascular death, MI, heart failure, and stroke during an average of 4.8 years of follow-up. ${ }^{111}$ The usefulness 
of sPLA $_{2}$ as a predictive biomarker has also been investigated in patients with ACS. A trial involving 1,032 ACS patients found a significant correlation between $\mathrm{SPA}_{2}$ mass and activity ( $\mathrm{r}=0.67)$, and an association between both measurements and the risk of cardiovascular events. ${ }^{105}$ Similar findings have also been reported in the French Registry of Acute ST Elevation or Non-ST-Elevation Myocardial Infarction (FAST-MI) trial. ${ }^{110}$ The plasma sPLA 2 level has also been shown to be an independent predictor of clinical coronary events in patients with unstable angina. ${ }^{109}$ In a 255-patient study, SPLA $_{2}$ levels were significantly higher in patients with unstable angina than in those with stable angina or healthy subjects, and had a significant correlation with a 2-year risk of developing clinical coronary events. ${ }^{109}$ Furthermore, the investigators of the 446-patient study called Global Registry of Acute Coronary Events (GRACE) reported that $\mathrm{sPLA}_{2}$ activity was associated with the risk of death and MI in a stepwise fashion with increasing tertiles of $\mathrm{SPLA}_{2} \cdot{ }^{107}$

Currently, no commercially available assay can quantify

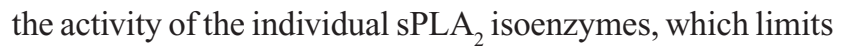
the use of $\mathrm{sPLA}_{2}$ in clinical practice. However, with a clear understanding of the molecular mechanism for its role in cardiovascular disease and a strong correlation with clinical outcomes in a variety of cardiovascular diseases, sPLA presents as a potentially highly useful endpoint biomarker in clinical research.

\section{GDF- I 5}

GDF-15 is a member of the transforming growth factor superfamily. GDF-15 is present in macrophages and many types of cardiovascular cells, and its expression is triggered by a variety of inflammatory mediators, such as oxidative stress, oxidized LDL, and various cytokines. ${ }^{112,113}$ Cardiomyocytes can also produce GDF-15 in response to these triggers, as well as mechanical stretch. ${ }^{112,114,115}$ Similarly, adipocytes are known to release GDF-15 in response to oxidative stress. ${ }^{116,117}$

While not much is known about its function, the pathophysiologic role of GDF-15 in cardiovascular disease is thought to be a part of the defensive mechanism by myocytes in response to ischemia or increased myocyte stress. Rapid elevation of GDF-15 in the ischemic area after coronary artery ligation was observed in murine models of ischemia and reperfusion injury lasting several days. ${ }^{12,118}$ Likewise, increased levels of GDF-15 are observed in patients with atherosclerosis. ${ }^{119}$ Furthermore, infusion of recombinant GDF-15 into infarcted myocardium suppresses the inflammatory response, suggesting a role as a counterregulatory cytokine. ${ }^{120}$
Increased levels of GDF-15 have been associated with increased overall and cardiovascular mortality in patients with ST segment elevation MI, ${ }^{121,122}$ non-ST segment elevation $\mathrm{MI},{ }^{123}$ and stable CHF. ${ }^{124}$ In three large clinical trials involving non-ST elevation-ACS patients (the GUSTO-IV, FRISC II, and Invasive versus Conservative Treatment in Unstable coronary Syndromes [ICTUS] sub-studies), higher GDF-15 level was independently associated with higher mortality rate over a 2-year follow-up period in the first two studies, and a 5-year follow-up period in the other. ${ }^{123,125,126}$ Furthermore, the FRISC II study found an association between elevated GDF-15 and increased rate of MI recurrence in patients with CAD ( $n=230)$, as well as the severity of coronary disease on angiogram.

While the role of GDF-15 in cardiovascular disease is clear, the utility of GDF-15 as a cardiovascular biomarker has not been so straightforward. In contrast to the GUSTO-IV, FRISC II, and ICTUS trials, the AtheroGene study found that patients with stable angina $(n=1,352)$ and ACS $(n=877)$ with GDF-15 greater than $1,800 \mathrm{ng} / \mathrm{L}$ are not at increased risk of nonfatal MI compared to those with low GDF-15. ${ }^{127}$ These patients were, however, at an increased risk of CHD mortality. The findings suggest that circulating GDF-15 levels are a stronger risk marker for mortality than for nonfatal coronary events in high-risk populations. In support of this notion, a 646-patient study called the Advantageous Predictors of Acute Coronary Syndrome Evaluation (APACE) study also found GDF-15 to be a better predictor of mortality than of nonfatal $\mathrm{CV}$ events. ${ }^{128}$ In another study involving patients with acute MI, GDF-15 was inferior to high-sensitivity troponin $\mathrm{T}$ and brain natriuretic peptide in detecting acute MI. ${ }^{128,129}$ However, GDF-15 was superior to both markers in predicting all-cause mortality. Furthermore, GDF-15 was as reliable as high-sensitivity troponin $\mathrm{T}$ in predicting death or future acute $\mathrm{MI}$ in patients presenting with chest pain without acute MI. The findings above raise questions as to whether GDF-15 can be a diagnostic tool for cardiovascular diseases. Even so, all evidence points to GDF-15 being a valuable biomarker in the context of cardiovascular medicine and warrants further research regarding its application in clinical practice and research.

\section{Conclusion}

The field of cardiovascular medicine has become a progressively competitive and costly field for the development of novel therapies and establishing clinical benefits over currently available therapies. Despite the challenges, cardiovascular disease is the leading health concern in the United States 
and warrants continued research and development for novel treatment options to address this still unmet need. To do so, biomarkers have become increasingly important in accurately assessing both the efficacy and safety of these novel therapies. Current established biomarkers, such as LDL-C and blood pressure, fail to fully capture and account for the entire cardiovascular disease process and to provide clear targets for lowering mortality. An undeniable need exists for novel biomarkers to fully understand the disease process and identify new treatment targets for the improvement of clinical outcomes in patients with cardiovascular disease. Significant efforts are underway to explore such novel biomarkers, including hs-CRP, PCSK9, Lp-PLA ${ }_{2}$, sPLA $_{2}$, and GDF-15. Whether the aforementioned biomarkers prove useful is still up to debate and more clinical trials are necessary to establish their validity. Nonetheless, new biomarkers are sure to play a critical role in both clinical practice and research, and a deep understanding of the benefits and limitations of these biomarkers will be crucial for clinicians and researchers alike.

\section{Disclosure}

The authors report no conflicts of interest in this work.

\section{References}

1. Murphy SL, Xu J, Kochanek KD. Deaths: Final Data for 2010. National Vital Statistics Reports; 2013. Available from: http://www.cdc.gov/nchs/ data/nvsr/nvsr61/nvsr61_04.pdf. Accessed February 1, 2014.

2. Biomarkers Definitions Working Group. Biomarkers and surrogate endpoints: preferred definitions and conceptual framework. Clin Pharmacol Ther. 2001;69(3):89-95.

3. Vasan RS. Biomarkers of cardiovascular disease: molecular basis and practical considerations. Circulation. 2006;113(19):2335-2362.

4. Vogel CL, Cobleigh MA, Tripathy D, et al. First-line Herceptin monotherapy in metastatic breast cancer. Oncology. 2001;61 Suppl 2:37-42.

5. Wilson PW, D'Agostino RB, Levy D, Belanger AM, Silbershatz H, Kannel WB. Prediction of coronary heart disease using risk factor categories. Circulation. 1998;97(18):1837-1847.

6. Straus SM1, Kors JA, De Bruin ML, et al. Prolonged QTc interval and risk of sudden cardiac death in a population of older adults. $J$ Am Coll Cardiol. 2006;47(2):362-367.

7. Yousuf O1, Mohanty BD, Martin SS, et al. High-sensitivity C-reactive protein and cardiovascular disease: a resolute belief or an elusive link? J Am Coll Cardiol. 2013;62(5):397-408.

8. National Cholesterol Education Program (NCEP) Expert Panel on Detection, Evaluation, and Treatment of High Blood Cholesterol in Adults (Adult Treatment Panel III). Third Report of the National Cholesterol Education Program (NCEP) Expert Panel on Detection, Evaluation, and Treatment of High Blood Cholesterol in Adults (Adult Treatment Panel III) final report. Circulation. 2002;106(25):3143-3421.

9. Ridker PM, Rifai N, Pfeffer MA, et al. Inflammation, pravastatin, and the risk of coronary events after myocardial infarction in patients with average cholesterol levels. Cholesterol and Recurrent Events (CARE) Investigators. Circulation. 1998;98(9):839-844.

10. Multiple risk factor intervention trial. Risk factor changes and mortality results. Multiple Risk Factor Intervention Trial Research Group. JAMA.1982;248:465-1477.
11. Ridker PM, Buring JE, Shih J, Matias M, Hennekens CH. Prospective study of C-reactive protein and the risk of future cardiovascular events among apparently healthy women. Circulation. 1998;98(8): 731-733.

12. Blaha MJ1, Rivera JJ, Budoff MJ, et al. Association between obesity, high-sensitivity C-reactive protein $>/=2 \mathrm{mg} / \mathrm{L}$, and subclinical atherosclerosis: implications of JUPITER from the Multi-Ethnic Study of Atherosclerosis. Arterioscler Thromb Vasc Biol. 2011;31(6): 1430-1438.

13. Ridker PM, Danielson E, Fonseca FA, et al; JUPITER Study Group. Rosuvastatin to prevent vascular events in men and women with elevated C-reactive protein. N Engl J Med. 2008;359(21):2195-2207.

14. Hingorani AD1, Sofat R, Morris RW, et al. Is it important to measure or reduce $\mathrm{C}$-reactive protein in people at risk of cardiovascular disease? Eur Heart J. 2012;33(18):2258-2264.

15. Sattar N, Murray HM, McConnachie A, et al; PROSPER Study Group. C-reactive protein and prediction of coronary heart disease and global vascular events in the Prospective Study of Pravastatin in the Elderly at Risk (PROSPER). Circulation. 2007;115(8):981-989.

16. Heart Protection Study Collaborative Group, Jonathan Emberson, Derrick Bennett, et al. C-reactive protein concentration and the vascular benefits of statin therapy: an analysis of 20,536 patients in the Heart Protection Study. Lancet. 2011;377(9764):469-476.

17. Woloshin S, Schwartz LM. Distribution of C-reactive protein values in the United States. N Engl J Med. 2005;352(15):1611-1613.

18. Harris TB, Ferrucci L, Tracy RP, et al. Associations of elevated interleukin-6 and C-reactive protein levels with mortality in the elderly. Am J Med. 1999;106(5):506-512.

19. Gupta NK, de Lemos JA, Ayers CR, Abdullah SM, McGuire DK, KheraA. The relationship between C-reactive protein and atherosclerosis differs on the basis of body mass index: the Dallas Heart Study. $J$ Am Coll Cardiol. 2012;60(13):1148-1155.

20. Bower JK, Lazo M, Juraschek SP, Selvin E. Within-person variability in high-sensitivity C-reactive protein. Arch Intern Med. 2012;172(19): 1519-1521.

21. Pearson TA, Blair SN, Daniels SR, et al. AHA Guidelines for Primary Prevention of Cardiovascular Disease and Stroke: 2002 Update: Consensus Panel Guide to Comprehensive Risk Reduction for Adult Patients Without Coronary or Other Atherosclerotic Vascular Diseases. American Heart Association Science Advisory and Coordinating Committee. Circulation. 2002;106(3):388-391.

22. Farnier M. PCSK9: From discovery to therapeutic applications. Arch Cardiovasc Dis. 2014;107(1):58-66.

23. Lose JM, Dorsch MP, Bleske BE. Evaluation of proprotein convertase subtilisin/kexin type 9: focus on potential clinical and therapeutic implications for low-density lipoprotein cholesterol lowering. Pharmacotherapy. 2013;33(4):447-460.

24. Folsom AR, Peacock JM, Boerwinkle E. Variation in PCSK9, low LDL cholesterol, and risk of peripheral arterial disease. Atherosclerosis. 2009;202(1):211-215.

25. Hallman DM, Srinivasan SR, Chen W, Boerwinkle E, Berenson GS. Relation of PCSK9 mutations to serum low-density lipoprotein cholesterol in childhood and adulthood (from The Bogalusa Heart Study). Am J Cardiol. 2007;100(1):69-72.

26. Careskey HE, Davis RA, Alborn WE, Troutt JS, Cao G, Konrad RJ. Atorvastatin increases human serum levels of proprotein convertase subtilisin/kexin type 9. J Lipid Res. 2008;49(2):394-398.

27. Awan Z, Seidah NG, MacFadyen JG, et al. Rosuvastatin, proprotein convertase subtilisin/kexin type 9 concentrations, and LDL cholesterol response: the JUPITER trial. Clin Chem. 2012;58(1):183-189.

28. Blann AD, Nadar SK, Lip GY. The adhesion molecule P-selectin and cardiovascular disease. Eur Heart J. 2003;24(24):2166-2179.

29. Nijmeijer R1, Meuwissen M, Krijnen PA, et al. Secretory type II phospholipase A2 in culprit coronary lesions is associated with myocardial infarction. Eur J Clin Invest. 2008;38(4):205-210.

30. Blann AD1, Lip GY. Hypothesis: is soluble P-selectin a new marker of platelet activation? Atherosclerosis. 1997;128(2):135-138. 
31. Woollard KJ1, Kling D, Kulkarni S, Dart AM, Jackson S, Chin-Dusting J. Raised plasma soluble P-selectin in peripheral arterial occlusive disease enhances leukocyte adhesion. Circ Res. 2006;98(1):149-156.

32. Galkina E, Ley K. Double jeopardy: how soluble P-selectin activates leukocytes in peripheral arterial occlusive disease. Circ Res. 2006;98(1): $12-14$.

33. Myers DD1, Hawley AE, Farris DM, et al. P-selectin and leukocyte microparticles are associated with venous thrombogenesis. JVasc Surg. 2003;38(5):1075-1089.

34. André P, Hartwell D, Hrachovinová I, Saffaripour S, Wagner DD. Pro-coagulant state resulting from high levels of soluble P-selectin in blood. Proc Natl Acad Sci U S A. 2000;97(25):13835-13840.

35. Blann A1, Morris J, McCollum C. Soluble L-selectin in peripheral arterial disease: relationship with soluble E-selectin and soluble P-selectin. Atherosclerosis. 1996;126(2):227-231.

36. Davì G, Romano M, Mezzetti A, et al. Increased levels of soluble P-selectin in hypercholesterolemic patients. Circulation. 1998;97(10): 953-957.

37. Lip GY1, Blann AD, Zarifis J, Beevers M, Lip PL, Beevers DG. Soluble adhesion molecule P-selectin and endothelial dysfunction in essential hypertension: implications for atherogenesis? A preliminary report. J Hypertens. 1995;13(12 Pt 2):1674-1678.

38. Ikeda H1, Takajo Y, Ichiki K, et al. Increased soluble form of P-selectin in patients with unstable angina. Circulation. 1995;92(7):1693-1696.

39. Kisucka J, Chauhan AK, Zhao BQ, et al. Elevated levels of soluble $\mathrm{P}$-selectin in mice alter blood-brain barrier function, exacerbate stroke, and promote atherosclerosis. Blood. 2009;113(23):6015-6022.

40. Badr Eslam R, Gremmel T, Schneller A, et al. High levels of plateletmonocyte aggregates after valve replacement for aortic stenosis: relation to soluble P-selectin and P-selectin glycoprotein ligand-1 genes. Thromb Res. 2012;129(4):453-458.

41. van Gils JM1, Zwaginga JJ, Hordijk PL. Molecular and functional interactions among monocytes, platelets, and endothelial cells and their relevance for cardiovascular diseases. J Leukoc Biol. 2009;85(2):195-204.

42. Myers DD Jr, Schaub R, Wrobleski SK, et al. P-selectin antagonism causes dose-dependent venous thrombosis inhibition. Thromb Haemost. 2001;85(3):423-429.

43. Oostingh GJ, Pozgajova M, Ludwig RJ, et al. Diminished thrombus formation and alleviation of myocardial infarction and reperfusion injury through antibody- or small-molecule-mediated inhibition of selectindependent platelet functions. Haematologica. 2007;92(4):502-512.

44. Sim D1, Flaumenhaft R, Furie B, Furie B. Interactions of platelets, blood-borne tissue factor, and fibrin during arteriolar thrombus formation in vivo. Microcirculation. 2005;12(3):301-311.

45. Wang J1, Zhang S, Jin Y, Qin G, Yu L, Zhang J. Elevated levels of platelet-monocyte aggregates and related circulating biomarkers in patients with acute coronary syndrome. Int J Cardiol. 2007;115(3): 361-365.

46. Freedman JE, Loscalzo J. Platelet-monocyte aggregates: bridging thrombosis and inflammation. Circulation. 2002;105(18):2130-2132.

47. Kling D, Stucki C, Kronenberg S, et al. Pharmacological control of platelet-leukocyte interactions by the human anti-P-selectin antibody inclacumab - preclinical and clinical studies. Thromb Res. 2013;131(5): $401-410$.

48. Liuzzo G. Atherosclerosis: an inflammatory disease. Rays. 2001;26(4): 221-230.

49. Weissberg PL, Bennett MR. Atherosclerosis - an inflammatory disease. N Engl J Med. 1999;340(24):1928-1929.

50. Williams KJ, Tabas I. Atherosclerosis - an inflammatory disease. NEngl J Med. 1999;340(24):1928; author reply 1929.

51. Sloop GD. Atherosclerosis - an inflammatory disease. N Engl J Med. 1999;340(24):1928; author reply 1929.

52. Ross R. Atherosclerosis - an inflammatory disease. $N$ Engl J Med. 1999;340(2):115-126.

53. Marecková Z, Heller S, Horký K. [Cell adhesion molecules and their role in pathophysiologic processes]. Vnitr Lek. 1999;45(1):46-50. Czech.
54. Golias C, Tsoutsi E, Matziridis A, Makridis P, Batistatou A, Charalabopoulos K. Review. Leukocyte and endothelial cell adhesion molecules in inflammation focusing on inflammatory heart disease. In Vivo. 2007;21(5):757-769.

55. Alfonso F, Angiolillo DJ. Targeting p-selectin during coronary interventions: the elusive link between inflammation and platelets to prevent myocardial damage. J Am Coll Cardiol. 2013;61(20):2056-2059.

56. Cambien B, Wagner DD. A new role in hemostasis for the adhesion receptor P-selectin. Trends Mol Med. 2004;10(4):179-186.

57. Johnson-Tidey RR, McGregor JL, Taylor PR, Poston RN. Increase in the adhesion molecule P-selectin in endothelium overlying atherosclerotic plaques. Coexpression with intercellular adhesion molecule-1. Am J Pathol. 1994;144(5):952-961.

58. Cassar K1, Bachoo P, Ford I, Greaves M, Brittenden J. Platelet activation is increased in peripheral arterial disease. J Vasc Surg. 2003;38(1): 99-103.

59. Zeiger F, Stephan S, Hoheisel G, Pfeiffer D, Ruehlmann C, Koksch M. P-Selectin expression, platelet aggregates, and platelet-derived microparticle formation are increased in peripheral arterial disease. Blood Coagul Fibrinolysis. 2000;11(8):723-728.

60. Gurbel PA, Kereiakes DJ, Dalesandro MR, Bahr RD, O’Connor CM, Serebruany VL. Role of soluble and platelet-bound P-selectin in discriminating cardiac from noncardiac chest pain at presentation in the emergency department. Am Heart J. 2000;139(2 Pt 1):320-328.

61. Tenaglia AN1, Buda AJ, Wilkins RG, et al. Levels of expression of P-selectin, E-selectin, and intercellular adhesion molecule-1 in coronary atherectomy specimens from patients with stable and unstable angina pectoris. Am J Cardiol. 1997;79(6):742-747.

62. Stellos K, Bigalke B, Stakos D, Henkelmann N, Gawaz M. Plateletbound $\mathrm{P}$-selectin expression in patients with coronary artery disease: impact on clinical presentation and myocardial necrosis, and effect of diabetes mellitus and anti-platelet medication. J Thromb Haemost. 2010;8(1):205-207.

63. Cao YJ1, Wang YM, Zhang J, Zeng YJ, Liu CF. The effects of antiplatelet agents on platelet-leukocyte aggregations in patients with acute cerebral infarction. J Thromb Thrombolysis. 2009;27(2):233-238.

64. Nadar SK, Lip GY, Blann AD. Platelet morphology, soluble P selectin and platelet P-selectin in acute ischaemic stroke. The West Birmingham Stroke Project. Thromb Haemost. 2004;92(6):1342-1348.

65. Yusuf SW, Sanderson H, Heptinstall S, Nurden AT, Wenham PW, Hopkinson BR. ADP-induced P-selectin expression on platelets as a predictor of successful thrombolysis. Blood Coagul Fibrinolysis. 1996;7(2):266-269.

66. Kayikçioglu M, Can L, Mete-Erdem N, et al. Soluble P-selectin and the success of thrombolysis in acute myocardial infarction. Int $J$ Cardiol. 2001;79(2-3):223-229.

67. Minamino T, Kitakaze M, Asanuma H, et al. Plasma adenosine levels and platelet activation in patients with atrial fibrillation. Am J Cardiol. 1999;83(2):194-198.

68. Shimomura H1, Ogawa H, Arai H, et al. Serial changes in plasma levels of soluble P-selectin in patients with acute myocardial infarction. Am J Cardiol. 1998;81(4):397-400.

69. Thomas R, Cheng Y, Yan J, et al. Upregulation of coronary endothelial $\mathrm{P}$-selectin in a monkey heart ischemia reperfusion model. J Mol Histol. 2010;41(4-5):277-287.

70. Hansen A1, Kumar A, Wolf D, et al. Evaluation of cardioprotective effects of recombinant soluble P-selectin glycoprotein ligandimmunoglobulin in myocardial ischemia-reperfusion injury by real-time myocardial contrast echocardiography. J Am Coll Cardiol. 2004;44(4): 887-891.

71. Wang K, Zhou X, Zhou Z, et al. Recombinant soluble P-selectin glycoprotein ligand-Ig (rPSGL-Ig) attenuates infarct size and myeloperoxidase activity in a canine model of ischemia-reperfusion. Thromb Haemost. 2002;88(1):149-154.

72. Hayward R, Campbell B, Shin YK, Scalia R, Lefer AM. Recombinant soluble P-selectin glycoprotein ligand-1 protects against myocardial ischemic reperfusion injury in cats. Cardiovasc Res. 1999;41(1):65-76. 
73. Downing LJ, Wakefield TW, Strieter RM, et al. Anti-P-selectin antibody decreases inflammation and thrombus formation in venous thrombosis. J Vasc Surg. 1997;25(5):816-827; discussion 828.

74. Wakefield TW, Strieter RM, Prince MR, Downing LJ, Greenfield LJ. Pathogenesis of venous thrombosis: a new insight. Cardiovasc Surg. 1997;5(1):6-15.

75. Wakefield TW, Strieter RM, Downing LJ, et al. P-selectin and TNF inhibition reduce venous thrombosis inflammation. J Surg Res. 1996;64(1):26-31.

76. Chen LY1, Nichols WW, Hendricks JB, Yang BC, Mehta JL. Monoclonal antibody to P-selectin (PB1.3) protects against myocardial reperfusion injury in the dog. Cardiovasc Res. 1994;28(9):1414-1422.

77. Bienvenu JG, Tanguay JF, Théorêt JF, Kumar A, Schaub RG, Merhi Y. Recombinant soluble p-selectin glycoprotein ligand-1-Ig reduces restenosis through inhibition of platelet-neutrophil adhesion after double angioplasty in swine. Circulation. 2001;103(8):1128-1134.

78. Kaufmann J, Wellnhofer E, Kappert K, et al. Soluble P-selectin level correlates with acetylsalicylic acid but not with clopidogrel response in patients with stable coronary artery disease after a percutaneous coronary intervention. Coron Artery Dis. 2013;24(4):312-320.

79. Day JR1, Malik IS, Weerasinghe A, et al. Distinct yet complementary mechanisms of heparin and glycoprotein IIb/IIIa inhibitors on platelet activation and aggregation: implications for restenosis during percutaneous coronary intervention. Heart. 2004;90(7):794-799.

80. Koenig A, Norgard-Sumnicht K, Linhardt R, Varki A. Differential interactions of heparin and heparan sulfate glycosaminoglycans with the selectins. Implications for the use of unfractionated and low molecular weight heparins as therapeutic agents. J Clin Invest. 1998;101(4): 877-889.

81. Xie X, Rivier AS, Zakrzewicz A, et al. Inhibition of selectin-mediated cell adhesion and prevention of acute inflammation by nonanticoagulant sulfated saccharides. Studies with carboxyl-reduced and sulfated heparin and with trestatin a sulfate. J Biol Chem. 2000;275(44):34818-34825.

82. Cha JK, Jeong MH, Kim JW. Statin reduces the platelet P-selectin expression in atherosclerotic ischemic stroke. J Thromb Thrombolysis. 2004;18(1):39-42.

83. Marschang P, Friedrich GJ, Ditlbacher H, et al. Reduction of soluble $\mathrm{P}$-selectin by statins is inversely correlated with the progression of coronary artery disease. Int J Cardiol. 2006;106(2):183-190.

84. Rosenson RS, Hurt-Camejo E. Phospholipase A2 enzymes and the risk of atherosclerosis. Eur Heart J. 2012;33(23):2899-2909.

85. Oörni K, Kovanen PT. Lipoprotein modification by secretory phospholipase A(2) enzymes contributes to the initiation and progression of atherosclerosis. Curr Opin Lipidol. 2009;20(5):421-427.

86. Zalewski A, Macphee C. Role of lipoprotein-associated phospholipase A2 in atherosclerosis: biology, epidemiology, and possible therapeutic target. Arterioscler Thromb Vasc Biol. 2005;25(5):923-931.

87. Stafforini DM, McIntyre TM, Carter ME, Prescott SM. Human plasma platelet-activating factor acetylhydrolase. Association with lipoprotein particles and role in the degradation of platelet-activating factor. $J$ Biol Chem. 1987;262(9):4215-4222.

88. Tellis CC, Tselepis AD. The role of lipoprotein-associated phospholipase A2 in atherosclerosis may depend on its lipoprotein carrier in plasma. Biochim Biophys Acta. 2009;1791(5):327-338.

89. De Keyzer D, Karabina SA, Wei W, et al. Increased PAFAH and oxidized lipids are associated with inflammation and atherosclerosis in hypercholesterolemic pigs. Arterioscler Thromb Vasc Biol. 2009;29(12): 2041-2046.

90. Packard CJ, O'Reilly DS, Caslake MJ, et al. Lipoprotein-associated phospholipase A2 as an independent predictor of coronary heart disease. West of Scotland Coronary Prevention Study Group. $N$ Engl J Med. 2000;343(16):1148-1155.

91. Brilakis ES, McConnell JP, Lennon RJ, Elesber AA, Meyer JG, Berger PB. Association of lipoprotein-associated phospholipase A2 levels with coronary artery disease risk factors, angiographic coronary artery disease, and major adverse events at follow-up. Eur Heart J. 2005;26(2):137-144.
92. Koenig W, Twardella D, Brenner H, Rothenbacher D. Lipoproteinassociated phospholipase A2 predicts future cardiovascular events in patients with coronary heart disease independently of traditional risk factors, markers of inflammation, renal function, and hemodynamic stress. Arterioscler Thromb Vasc Biol. 2006;26(7):1586-1593.

93. Corsetti JP, Rainwater DL, Moss AJ, Zareba W, Sparks CE. High lipoprotein-associated phospholipase A2 is a risk factor for recurrent coronary events in postinfarction patients. Clin Chem. 2006;52(7): $1331-1338$.

94. Zheng D, Zeng F, Cai A, et al. Baseline elevated Lp-PLA2 is associated with increased risk for re-stenosis after stent placement. Lipids Health Dis. 2014;13:41.

95. Möckel M, Müller R, Vollert J, et al. Lipoprotein-associated phospholipase A2 for early risk stratification in patients with suspected acute coronary syndrome: a multi-marker approach: the North Wuerttemberg and Berlin Infarction Study-II (NOBIS-II). Clin Res Cardiol. 2007;96(9):604-612.

96. GerberY, McConnell JP, Jaffe AS, Weston SA, Killian JM, Roger VL. Lipoprotein-associated phospholipase A2 and prognosis after myocardial infarction in the community. Arterioscler Thromb Vasc Biol. 2006;26(11):2517-2522.

97. Oldgren J, James SK, Siegbahn A, Wallentin L. Lipoprotein-associated phospholipase A2 does not predict mortality or new ischaemic events in acute coronary syndrome patients. Eur Heart J. 2007;28(6): 699-704.

98. O’Donoghue M, Morrow DA, Sabatine MS, et al. Lipoproteinassociated phospholipase A2 and its association with cardiovascular outcomes in patients with acute coronary syndromes in the PROVE IT-TIMI 22 (PRavastatin Or atorVastatin Evaluation and Infection Therapy-Thrombolysis In Myocardial Infarction) trial. Circulation. 2006;113(14):1745-1752.

99. STABILITY Investigators, White HD, Held C, et al. Darapladib for preventing ischemic events in stable coronary heart disease. $\mathrm{N} \mathrm{Engl}$ J Med. 2014;370(18):1702-1711.

100. GlaxoSmithKline. GSK announces phase III study with darapladib did not meet primary endpoint in patients following an acute coronary syndrome [press release]. Brentford: GlaxoSmithKline; May 13, 2014. Available from: http://www.gsk.com/media/press-releases/2014/gskannounces-phase-iii-study-with-darapladib-did-not-meet-prima.html. Accessed May 20, 2014.

101. Ivandic B, Castellani LW, Wang XP, et al. Role of group II secretory phospholipase A2 in atherosclerosis: 1. Increased atherogenesis and altered lipoproteins in transgenic mice expressing group IIa phospholipase A2. Arterioscler Thromb Vasc Biol. 1999;19(5):1284-1290.

102. Mallat Z, Benessiano J, Simon T, et al. Circulating secretory phospholipase A2 activity and risk of incident coronary events in healthy men and women: the EPIC-Norfolk study. Arterioscler Thromb Vasc Biol. 2007;27(5):1177-1183.

103. Boekholdt SM, Keller TT, Wareham NJ, et al. Serum levels of type II secretory phospholipase A2 and the risk of future coronary artery disease in apparently healthy men and women: the EPICNorfolk Prospective Population Study. Arterioscler Thromb Vasc Biol. 2005;25(4):839-846.

104. Kugiyama K, Ota Y, Takazoe K, et al. Circulating levels of secretory type II phospholipase A(2) predict coronary events in patients with coronary artery disease. Circulation. 1999;100(12):1280-1284.

105. Liu PY, Li YH, Tsai WC, et al. Prognostic value and the changes of plasma levels of secretory type II phospholipase A2 in patients with coronary artery disease undergoing percutaneous coronary intervention. Eur Heart J. 2003;24(20):1824-1832.

106. Sabatine MS, Morrow DA, O’Donoghue M, et al. Prognostic utility of lipoprotein-associated phospholipase A2 for cardiovascular outcomes in patients with stable coronary artery disease. Arterioscler Thromb Vasc Biol. 2007;27(11):2463-2469.

107. Mallat Z, Steg PG, Benessiano J, et al. Circulating secretory phospholipase A2 activity predicts recurrent events in patients with severe acute coronary syndromes. J Am Coll Cardiol. 2005;46(7):1249-1257. 
108. Koenig W, Vossen CY, Mallat Z, Brenner H, Benessiano J, Rothenbacher D. Association between type II secretory phospholipase A2 plasma concentrations and activity and cardiovascular events in patients with coronary heart disease. Eur Heart J. 2009;30(22): $2742-2748$.

109. Kugiyama K, Ota Y, Sugiyama S, et al. Prognostic value of plasma levels of secretory type II phospholipase A2 in patients with unstable angina pectoris. Am J Cardiol. 2000;86(7):718-722.

110. Lind L, Simon T, Johansson L, et al. Circulating levels of secretoryand lipoprotein-associated phospholipase A2 activities: relation to atherosclerotic plaques and future all-cause mortality. Eur Heart J. 2012;33(23):2946-2954.

111. O’Donoghue ML, Mallat Z, Morrow DA, et al. Prognostic utility of secretory phospholipase $\mathrm{A}(2)$ in patients with stable coronary artery disease. Clin Chem. 2011;57(9):1311-1317.

112. KempfT, Eden M, Strelau J, et al. The transforming growth factor-beta superfamily member growth-differentiation factor- 15 protects the heart from ischemia/reperfusion injury. Circ Res. 2006;98(3):351-360.

113. Bermúdez B, López S, Pacheco YM, et al. Influence of postprandial triglyceride-rich lipoproteins on lipid-mediated gene expression in smooth muscle cells of the human coronary artery. Cardiovasc Res. 2008;79(2):294-303.

114. Frank D, Kuhn C, Brors B, et al. Gene expression pattern in biomechanically stretched cardiomyocytes: evidence for a stretch-specific gene program. Hypertension. 2008;51(2):309-318.

115. Xu J, Kimball TR, Lorenz JN, et al. GDF15/MIC-1 functions as a protective and antihypertrophic factor released from the myocardium in association with SMAD protein activation. Circ Res. 2006;98(3): $342-350$

116. Kim JH, Kim KY, Jeon JH, et al. Adipocyte culture medium stimulates production of macrophage inhibitory cytokine 1 in MDA-MB-231 cells. Cancer Lett. 2008;261(2):253-262.

117. Ding Q, Mracek T, Gonzalez-Muniesa P, et al. Identification of macrophage inhibitory cytokine-1 in adipose tissue and its secretion as an adipokine by human adipocytes. Endocrinology. 2009;150(4): 1688-1696.

118. Bootcov MR, Bauskin AR, Valenzuela SM, et al. MIC-1, a novel macrophage inhibitory cytokine, is a divergent member of the TGF-beta superfamily. Proc Natl Acad Sci U S A. 1997;94(21):11514-11519.

119. Schlittenhardt D, Schober A, Strelau J, et al. Involvement of growth differentiation factor-15/macrophage inhibitory cytokine-1 (GDF-15/ MIC-1) in oxLDL-induced apoptosis of human macrophages in vitro and in arteriosclerotic lesions. Cell Tissue Res. 2004;318(2): 325-333.
120. Kempf T, Zarbock A, Widera C, et al. GDF-15 is an inhibitor of leukocyte integrin activation required for survival after myocardial infarction in mice. Nat Med. 2011;17(5):581-588.

121. Kempf T, Björklund E, Olofsson S, et al. Growth-differentiation factor-15 improves risk stratification in ST-segment elevation myocardial infarction. Eur Heart J. 2007;28(23):2858-2865.

122. Khan SQ, $\mathrm{Ng} \mathrm{K}$, Dhillon O, et al. Growth differentiation factor- 15 as a prognostic marker in patients with acute myocardial infarction. Eur Heart J. 2009;30(9):1057-1065.

123. Wollert KC, Kempf T, Peter T, et al. Prognostic value of growthdifferentiation factor-15 in patients with non-ST-elevation acute coronary syndrome. Circulation. 2007;115(8):962-971.

124. Kempf T, von Haehling S, Peter T, et al. Prognostic utility of growth differentiation factor-15 in patients with chronic heart failure. $\mathrm{J} \mathrm{Am}$ Coll Cardiol. 2007;50(11):1054-1060.

125. Wollert KC, Kempf T, Lagerqvist B, et al. Growth differentiation factor 15 for risk stratification and selection of an invasive treatment strategy in non ST-elevation acute coronary syndrome. Circulation. 2007;116(14):1540-1548.

126. Damman P, Kempf T, Windhausen F, et al; ICTUS investigators. Growth-differentiation factor 15 for long-term prognostication in patients with non-ST-elevation acute coronary syndrome: An Invasive versus Conservative Treatment in Unstable coronary Syndromes (ICTUS) substudy. Int J Cardiol. 2014;172(2):356-363.

127. KempfT, Sinning JM, Quint A, et al. Growth-differentiation factor-15 for risk stratification in patients with stable and unstable coronary heart disease: results from the AtheroGene study. Circ Cardiovasc Genet. 2009;2(3):286-292.

128. Schaub N, Reichlin T, Twerenbold R, et al. Growth differentiation factor-15 in the early diagnosis and risk stratification of patients with acute chest pain. Clin Chem. 2012;58(2):441-449.

129. Rohatgi, A. Usefulness of Growth Differentiation Factor-15 (GDF-15) As A Marker for Patients with Possible MI and For Predicting Coronary Disease in Asymptomatic Patients. Cardiac Biomarkers (2012) http:// biomarkers.cardiosource.org/Hot-Topics/2012/02/Using-GDF-15-asa-Marker-for-Predicting-Coronary-Disease.aspx. Accessed March 20, 2014.
Current Biomarker Findings

\section{Publish your work in this journal}

Current Biomarker Findings is an international, peer-reviewed, open access journal publishing original research, reports, reviews and commentaries on all areas of biomarker research. The manuscript management system is completely online and includes a very quick and fair

\section{Dovepress}

peer-review system. Visit http://www.dovepress.com/testimonials.php to read real quotes from published authors. 